\title{
Langfristige Wirkung von Psychotherapie bei nichtchronischen Depressionen: Ein systematisches Review von Studien im Vergleich mit Pharmakotherapie
}

\author{
Ulrich Voderholzer ${ }^{a, b} \quad$ Barbara Barton ${ }^{c}$ \\ ${ }^{a}$ Klinik für Psychiatrie und Psychotherapie, Universitätsklinikum Freiburg, Freiburg, Deutschland; \\ bSchön Klinik Roseneck, Prien am Chiemsee, Deutschland; \\ ${ }^{c}$ Klinik für Psychiatrie und Psychotherapie, Universitätsklinikum München, München, Deutschland
}

\section{Schlüsselwörter}

Depression · Psychotherapie - Langzeitwirkungen . Antidepressiva

\section{Zusammenfassung}

Die kurzfristige Wirksamkeit von Psycho- und Pharmakotherapie bei Depressionen, ob als Monotherapie oder in Kombination, ist durch viele Studien gut belegt. Weitaus weniger Belege gibt es jedoch für die langfristige Wirksamkeit ab 1 Jahr nach Akuttherapie dieser Behandlungsformen. Durch eine systematische Suche in den Datenbanken Pubmed und PsychINFO konnten 13 randomisierte kontrollierte Studien (RCTs; Psychotherapie vs. Pharmakotherapie in Kombination oder als Monotherapien) identifiziert werden, bei denen Follow-up-Ergebnisse vorliegen; der entsprechende Follow-up-Zeitraum lag dabei zwischen 13 und 75 Monaten ( $M=27,06)$. Meist kam als psychotherapeutisches Verfahren die kognitive Verhaltenstherapie zum Einsatz. Die Studienlage spricht für eine Überlegenheit der Psychotherapie oder der Kombination aus Psychotherapie und Pharmakotherapie im Vergleich zur Pharmakotherapie mit oder ohne Erhaltungsphase nach der Akutbehandlung bezüglich langfristiger Effekte über die Beendigung der Therapie hinaus. Die Studien sind aufgrund vielfältiger methodischer Faktoren nur begrenzt vergleichbar (z.B. hinsichtlich der Datenselektion zu Patienten mit und ohne Therapieerfolg nach Akuttherapie oder weiterer Behandlungen im Follow-up-Zeitraum). Um besser abgesicherte Aussagen zur langfristigen Wirksamkeit von Psycho- und Pharmakotherapie in der Behandlung der Depression treffen zu können, bedarf es weiterer RCTs mit längeren Follow-up-Zeiträumen.

(c) 2016 S. Karger GmbH, Freiburg
Keywords

Depression · Psychotherapy · Long-term effects .

Antidepressant

\section{Summary}

The short-term effects of psychotherapy and pharmacotherapy - as monotherapy or in combination - in the treatment of depression have been documented in various studies. Strikingly, there are only a few studies proving the long-term efficacy 1 year or more after acute treatment. Therefore, a systematic review was conducted searching the databases Pubmed and PsychINFO. 13 randomized controlled trials (RCTs) could be retrieved (psychotherapy vs. pharmacotherapy in combination or as monotherapies). The follow-up period varied between 12 and 75 months ( $M=27.06)$, cognitive behavioral therapy being the most frequently used psychotherapeutic strategy. The results indicate an advantage of psychotherapy alone or the combination of psychotherapy and pharmacotherapy when compared with pharmacotherapy alone. The advantage persisted even if the pharmacotherapy was maintained during the follow-up period. However, comparing studies is difficult due to methodological issues (e.g., selection of data relating to patients with or without therapy success after acute therapy; further treatments during follow-up). In order to make more valid statements about the long-term efficacy of psychotherapy and pharmacotherapy in the treatment of depression, more RCTs with longer follow-up periods are needed.

\section{KARGER \\ Fax +497614520714




\section{Einleitung}

Depressive Erkrankungen haben aufgrund ihrer LebenszeitPrävalenz von 16,2\% [Kessler et al., 2003] und den mit der Erkrankung verbundenen hohen negativen sozioökonomischen Folgen [Marschall et al., 2016] eine große gesellschaftliche Bedeutung. Zu den Charakteristika depressiver Erkrankungen zählt ihre Rezidivneigung sowie bei etwa einem Drittel der Patienten auch eine Chronizität [Murphy und Byrne, 2012]. Singuläre depressive Episoden stellen eher die Ausnahme dar. So hatten in einer populationsbasierten Studie von Eaton et al. [2008] 15\% der Patienten innerhalb von 23 Jahren kein Jahr ohne depressive Episode und etwa die Hälfte der Patienten nach der ersten depressiven Episode mindestens eine weitere im Verlauf. Deshalb stellt neben der kurzfristigen Wirksamkeit nach Akutbehandlung auch die Langzeitwirksamkeit von Therapien im Sinne langfristiger Effekte und einer Senkung des Rezidivrisikos eine entscheidende therapeutische Herausforderung dar. In den gegenwärtigen Leitlinienempfehlungen [APA, 2010; DGPPN et al., 2015; NICE, 2016], bei denen eine Graduierung der Evidenz eine wesentliche Rolle bei der Bewertung des Wissenstands spielt, findet die Frage der langfristigen bzw. nachhaltigen Wirkung von Therapien nur wenig Berücksichtigung. Beispielsweise werden Empfehlungen vor allem dann ausgesprochen, wenn die Wirksamkeit der Akutbehandlung (Psychotherapie/ Pharmakotherapie) sehr gut belegt ist, z.B. durch mehrere randomisierte kontrollierte Studien (RCTs), meist mit einer Dauer von 6 oder 12 Wochen. Dies ist nicht unkritisch, da infrage gestellt werden kann, ob eine Therapie zu empfehlen ist, deren kurzfristige Wirksamkeit zwar sehr gut belegt ist, bei der aber unter Umständen eine gewisse Unsicherheit besteht, welche langfristigen Effekte - eventuell auch negativer Art - sie für den Betroffenen haben könnte. So gibt es einige Hinweise darauf, dass Antidepressiva langfristig auch ungünstige Effekte auf den Verlauf und das Rezidivrisiko bei Depressionen haben könnten [Andrews et al., 2012; Babyak et al., 2000; Shrestha et al., 2014]. Vor diesem Hintergrund ist es von besonderem Interesse, ob die Psychotherapie, deren kurzfristige Wirksamkeit bei Depressionen [Kognitive Verhaltenstherapie (KVT): Cuijpers et al., 2013a; Interpersonelle Psychotherapie (IPT): Cuijpers et al., 2011b; psychodynamische Therapie: Driessen et al., 2015] ebenso belegt ist wie für Antidepressiva, positive langfristige Effekte aufweist. Weiterhin ist die akute Wirksamkeit von Psychotherapie und medikamentöser Behandlung vergleichbar, wobei die Kombination aus beiden der jeweiligen alleinigen Anwendung überlegen zu sein scheint - dies gilt für alle Schweregrade der Depression [Cuijpers et al., 2011a; Cuijpers et al., 2014b; Weitz et al., 2015]. Etwa zwei Drittel der Patienten erfüllen nach einer Psychotherapie nicht mehr die Kriterien einer klinischen Depression [Cuijpers et al., 2014a]. Allerdings können die sehr guten Akuteffekte antidepressiver Therapieverfahren zu einem erheblichen Teil durch Placeboeffekte erklärt werden - dies gilt sowohl für Antidepressiva als auch für die Psychotherapie [Khan et al., 2012; Kirsch, 2016]. Wie lange der Effekt der Therapie über deren Beendigung hinaus anhält (carry-over effect), ist allerdings weitaus weniger gut untersucht. Allgemein ist anerkannt, dass eine medikamentöse Therapie nach Akutbehandlung im Sinne einer Erhaltungstherapie zur Reduktion des Rückfallrisikos fortgeführt werden sollte. Leitlinien empfehlen deshalb die Weiterbehandlung mit Antidepressiva für mindestens 6 Monate nach Remissionseintritt [APA, 2010; DGPPN et al., 2015; NICE, 2016]. Die Psychotherapie hingegen wird in der Praxis entsprechend den Vorgaben der Richtlinien-Psychotherapie nach Aufbrauchen eines Stundenkontingents beendet. Eine Erhaltungstherapie der Psychotherapie würde auch nicht dem Verständnis von Psychotherapie entsprechen, die dem Patienten ja Fertigkeiten und Strategien vermitteln soll, die ihm helfen, seine Erkrankung zu bewältigen und künftig mit Triggerfaktoren anders umzugehen. In der Psychotherapie soll der Patienten also etwas lernen, das ihm auch nach Ende der Therapie anhaltend hilft und nicht abhängig von der Therapie macht. Bei diesem Konzept von Psychotherapie wird folglich von einem längerfristigen und rückfallprophylaktischen Effekt ausgegangen. Dies gilt für verhaltenstherapeutische wie psychodynamische Verfahren gleichermaßen. Dass sich die Praxis vielfach anders gestaltet und auch viele Betroffene eine Fortführung ihrer Therapie wünschen, weiß jeder Praktiker, aber auch in der Psychotherapieforschung wird das Thema Booster-Sitzungen, d.h. das Wiederauffrischen des in der Therapie Erlernten, schon lange diskutiert [Vittengl et al., 2007].

Langzeiteffekte der Behandlung der akut episodischen unipolaren Depression in bisherigen Meta-Analysen

In einer bereits vor 18 Jahren publizierten Meta-Analyse von Gloaguen et al. [1998] zur Effektivität von Kognitiver Therapie bei Depression gab es unter 48 RCTs nur 8 mit Follow-up-Daten von 1-2 Jahren. Bei 5 der 8 Studien zeigte sich eine langfristige Überlegenheit der Psycho- im Vergleich zur Pharmakotherapie: Die durchschnittlichen Rückfallraten zum Follow-up-Messpunkt lagen nach einer Kognitiven Therapie bei 29,5\% und nach einer Behandlung mit Antidepressiva bei $60 \%$. In einer Meta-Analyse von Vittengl et al. [2007] mit 28 Studien ( $=1880$ Patienten) zeigte sich, dass nach einer Akuttherapie mit Kognitiver Therapie bei Depressionen 29\% der Teilnehmer innerhalb 1 und 54\% innerhalb von 2 Jahren rückfällig wurden. Dabei wurden nur die Daten von Patienten mit Therapieerfolg nach der Akuttherapie berücksichtigt. Im Vergleich zur Pharmakotherapie reduziert die Kognitive Therapie das Rückfallrisiko jedoch um 22-23\%. Des Weiteren berichten die Autoren, dass die Rückfallrate mit größerer Dauer des Follow-up nach der Akuttherapie ansteigt. Letzteres konnten Steinert et al. [2014] nicht bestätigen. In 6 der in die Meta-Analyse von Steinert et al. [2014] eingeflossenen Studien wurde die Psychotherapie mit einem nichtpsychotherapeutischen Verfahren (Pharmakotherapie, Clinical Management, Treatment as Usual) verglichen. In den Studien wurden sowohl Patienten mit und ohne Therapieerfolg nach der Akutbehandlung eingeschlossen als auch solche mit depressiver Restsymptomatik. Es zeigte sich eine Überlegenheit der psychotherapeutischen Behandlung in einem Zeitraum von mehr als 2 Jahren: 53\% der Patienten in der psychotherapeutischen Behandlung und $71 \%$ in der nichtpsychotherapeutischen Behandlung hatten einen Rückfall. Weiterhin schließen die Autoren aus ihren Er- 
gebnissen, dass irgendeine Form von Psychotherapie, ob als Monooder kombinierte Therapie, das Rückfallrisiko in einer Zeitspanne von etwa 4,4 Jahren senkt. Zu ähnlichen Ergebnissen kamen Karyotaki et al. [2016] in einer Meta-Analyse mit 23 RCTs ( $N=2184$ Patienten). Die Kombinationsbehandlung aus Psycho- und Pharmakotherapie wurde hier mit den jeweiligen Monotherapien verglichen und zwar jeweils als Akuttherapie sowie als Erhaltungstherapie. Es wurden Studien mit und ohne Therapieerfolg nach der Akuttherapie als Einschlusskriterium eingeschlossen. Die Kombinationsbehandlung aus Psychotherapie und Pharmakotherapie sowie die alleinige Psychotherapie waren der alleinigen Pharmakotherapie überlegen. Bei einem Vergleich der Follow-up-Daten nach KVT und medikamentöser Erhaltungstherapie über 6-18 Monate zeigte sich in der Meta-Analyse von Cuijpers et al. [2013a] ein nichtsignifikanter Trend zugunsten der KVT. Der Vergleich der Nachhaltigkeit von Psychotherapie mit den Studienarmen, in denen zunächst Antidepressiva gegeben, diese dann jedoch abgesetzt wurden, ergab dagegen eine signifikante Überlegenheit zugunsten der KVT. In einer weiteren Meta-Analyse von Cuijpers et al. [2014b] mit 32 Studien ging es um den Vergleich einer Kombinationsbehandlung aus Psycho- und Pharmakotherapie mit alleiniger Pharmakotherapie. Dabei zeigte sich nach Akuttherapie und bis $\mathrm{zu} 2$ Jahre später eine signifikante Überlegenheit der Kombinationsbehandlung.

Die Ergebnisse bisheriger Meta-Analysen offenbaren, dass selbst Patienten, die auf eine psychotherapeutische Akutbehandlung ansprechen, in etwa der Hälfte der Fälle innerhalb von 2 Jahren wieder einen Rückfall erleiden und offensichtlich eine intensivere Rückfallprophylaxe benötigt wird. Weiterhin sind die Ergebnisse bisheriger Übersichtsarbeiten und auch Meta-Analysen bezüglich der Frage zur Langzeitwirksamkeit der Psychotherapie nur beschränkt aussagekräftig, da es bei den berücksichtigten Studien viele methodische Schwierigkeiten und Unterschiede gibt. So wurde oft nicht zwischen akuten und residualen depressiven Symptomen unterschieden oder es wurden nur Patienten mit Therapieerfolg in die Analyse eingeschlossen. Insbesondere ist diesen Studien die unzureichende Dokumentation im Follow-up-Zeitraum anzumerken. So wurde bei den Vergleichen häufig nicht unterschieden, ob nach Akutbehandlung eine Therapie fortgeführt wurde oder nicht. Ein fairer Vergleich von Behandlungsformen zur Beurteilung von Nachhaltigkeit setzt voraus, dass die Behandlungen nicht weitergeführt werden, sonst untersucht man nicht die Nachhaltigkeit, d.h. einen "carry-over effect», sondern die Frage einer «anhaltenden Wirkung». Das Ziel dieses systematischen Reviews war es deshalb, bisherige Langzeitstudien zur Behandlung der akut episodischen unipolaren Depression differenziert zu untersuchen, um mehr über die langfristige Wirksamkeit im Sinne des «carry-over effects» der psychotherapeutischen Akuttherapie im Vergleich zur medikamentösen Akuttherapie mit und ohne Erhaltungstherapie sowie zur Kombinationsbehandlung aus Psychotherapie und Pharmakotherapie zu erfahren. Das Thema «chronische Depression» wird in diesem Artikel nicht berücksichtigt; jedoch sei angemerkt, dass Langzeitstudien diesbezüglich sehr limitiert sind.

\section{Methodik}

\section{Suchstrategie}

Die Datenbanken Pubmed und PsychINFO wurden auf englisch- und deutschsprachige Artikel im Februar 2016 mit folgenden Suchbegriffen durchsucht: («long-term» [title] OR «follow-up» [title] OR «enduring effect» [title] OR «lasting effect» [title] OR «persist ${ }^{*} »$ [title] OR relapse [title] OR stability [title] OR stable [title] OR recurrence [title] OR relapse [title]) AND (depressi* [title] OR «affective disorder» [title] OR «depressive episode» [title] OR «major depression» [title]) AND (efficac ${ }^{\star}$ OR effect* OR outcome OR results) AND (treatment OR therapy OR CBT OR «cognitive behavioral therapy» OR IPT OR «interpersonal therapy» OR CBASP OR psychoanal ${ }^{\star}$ OR psychodynamic ${ }^{\star}$ OR psychotherapy ${ }^{\star}$ ) AND (antidepress ${ }^{\star}$ OR SSRI OR «Selective serotonin reuptake inhibitors» OR SNRI «Serotonin-norepinephrine reuptake inhibitors» $\mathrm{OR}$ «MAO inhibitors» $\mathrm{OR}$ «Monoamine oxidase inhibitors» OR NARI OR «noradrenaline reuptake inhibitors» OR «tricyclic antidepressants» OR TCA OR «Tetracyclic antidepressants» OR TeCA OR pharmacothera*). Es wurden nur Artikel von 1980-2016 berücksichtigt. Zusätzlich wurden die Literaturverzeichnisse der eingeschlossenen Studien sowie andere systematische Reviews und Meta-Analysen nach weiteren geeigneten Artikeln durchsucht.

\section{Ein-und Ausschlusskriterien}

Die Einschlusskriterien umfassten: 1) RCTs, 2) eine unipolare depressive Episode als Hauptdiagnose zu Beginn der Akuttherapie, 3) einen direkten Vergleich von Psychotherapie und Antidepressiva nach der Akuttherapie und nach dem Follow-up, 4) einen Follow-up-Zeitraum von mindestens 12 Monaten und 5) ein Erwachsenenalter $\geq 18$ Jahre. Ausgeschlossen wurden: 1) unkontrollierte Studien, 2) Studien, in denen im Follow-up-Zeitraum eine psychotherapeutische Erhaltungstherapie durchgeführt wurde und die somit das Einschlusskriterium des Zeitraums von 12 Monaten nicht mehr erfüllten, 3) Studien, in denen der Fokus auf der Reduktion depressiver Restsymptomatik lag, sowie 4) Studien mit Patienten mit Depression im Alter. Studien mit psychotherapeutischer Erhaltungstherapie, die jedoch nur solange dauerte, dass anschließend noch mindestens ein 12-monatiges Follow-up ohne Therapie folgte, wurden eingeschlossen.

\section{Ergebnisse}

Durch die Suchstrategie wurden 432 Studien identifiziert und gescreent. Insgesamt erfüllten 13 Studien mit 946 Patienten alle Einschlusskriterien und wurden in das systematische Review eingeschlossen (Abb.1, Online Supplemental Tabelle; www.karger. $\mathrm{com} /$ ?DOI=446674).

\section{Follow-up-Dauer}

Der Follow-up-Zeitraum in den hier betrachteten Studien lag zwischen 12 und 75 Monaten (M = 27,06 Monate). Die am häufigs- 


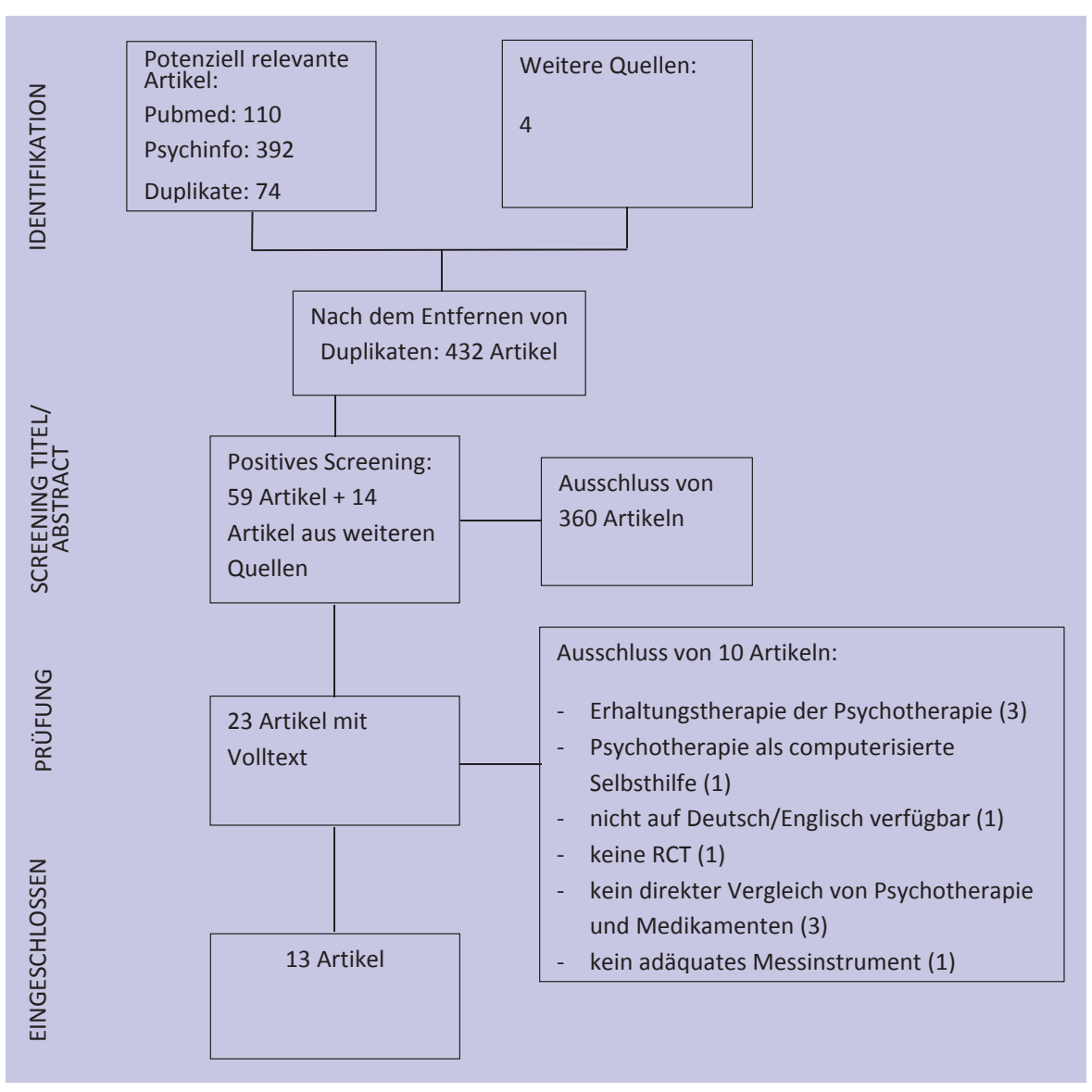

Abb. 1. Flow-Chart der eingeschlossenen Studien.

ten berichteten Follow-up-Zeiträume betrugen 12 und 24 Monate. Dieser Zeitraum wurde in 11 Studien untersucht, während ein Follow-up von mehr als 24 Monaten nur in 4 Studien durchgeführt wurde. Durchschnittlich bezogen sich die Daten auf Patienten mit einer mittelgradigen depressiven Episode (gemessen mit der Hamilton Depression Scale mit 17 Items; HAMD-17), bzw. knapp an der Grenze zur schweren depressiven Episode (gemessen mit dem Beck Depression Inventory; BDI).

\section{Psychotherapeutische Verfahren in der Akuttherapie}

Mehrheitlich wurden kognitive Verfahren untersucht. In der Hälfte der Studien wurde die Kognitive Therapie [Dobson et al., 2008; Blackburn et al., 1986; Evans et al., 1992; Simons et al., 1986; Beck et al., 1985; Kovacs et al., 1981], bei Shea et al. [1992] und De Jong-Meyer et al. [1996] die KVT und bei Dobson et al. [2008] die Verhaltensaktivierung untersucht. Weitere Therapieverfahren waren ein Problemlösetraining [Mynors-Wallis et al., 2000], die IPT [Schramm et al., 2007; Shea et al., 1992; Weissman et al., 1981; Zobel et al., 2011] sowie psychodynamische Therapieformen [Koppers et al., 2011; Maina et al., 2009]. Die Anzahl der Sitzungen lag zwischen 6-30 in einem Zeitraum von 5-24 Wochen.

\section{Antidepressiva in der Akuttherapie}

Das am häufigsten verwendete Antidepressivum war das trizyklische Antidepressivum Amitriptylin. Die Angaben in der Tabelle
(Online Supplemental Tabelle; www.karger.com/?DOI=446674) berücksichtigen auch die im Falle der Unverträglichkeit im Studienprotokoll aufgelisteten Medikamente.

\section{Behandlungssetting}

Bis auf 2 Ausnahmen [De Jong-Meyer et al., 1996; Schramm et al., 2007; Zobel et al., 2011] wurden die Akuttherapien ambulant durchgeführt.

Unterscheidung zwischen Patienten mit und ohne Therapieerfolg nach Akuttherapie

Die eingeschlossenen Studien unterschieden sich hinsichtlich der Einschlusskriterien für die Follow-up-Messung. So wurden in 4 Studien nur Patienten in die Follow-up-Messung aufgenommen, die nach Akuttherapie einen Therapieerfolg erzielten [Blackburn et al., 1986; Dobson et al., 2008; Evans et al., 1992; Maina et al., 2009]. Bei De Jong-Meyer et al. [1996], Weissman et al. [1981], Zobel et al. [2011], Shea et al. [1992] und Simons et al. [1986] gab es keine Ausschlusskriterien für den Follow-up-Zeitraum, wobei in den beiden letztgenannten Studien der Fokus der Datendarstellung auf Patienten mit Therapieerfolg lag. In weiteren 4 Studien wurden nur Completer der Akuttherapie in das Follow-up zugelassen [Beck et al., 1985; Koppers et al., 2011; Kovacs et al., 1981; MynorsWallis et al., 2000]. Bei Schramm et al. [2007] wurden die Intention-to-Treat(ITT)- und Completer-Analysen zu allen Messzeitpunkten differenziert dargestellt. 


\section{Angaben über Behandlungen der Depression innerhalb des} Follow-up-Zeitraums

In den meisten Fällen handelte es sich um ein naturalistisches Follow-up. Darunter wird verstanden, dass die Patienten nicht verpflichtet wurden, weiterführende Behandlungen oder die Wiederaufnahme von Behandlungen bezüglich der Depression zu unterlassen. In 5 Studien wurde die Weiterbehandlung der Depression im Follow-up -Zeitraum erfasst. In den meisten Fällen (14-91\%) kam es jedoch zu einer weiteren Behandlung während des Followup (Online Supplemental Tabelle; www.karger.com/?DOI=446674). Bei Shea et al. [1992], Beck et al. [1985] und bei Weissman et al. [1981] fällt auf, dass medikamentös behandelte Patienten im Follow-up-Zeitraum öfter weitere Therapien in Anspruch nahmen (Online Supplemental Tabelle; www.karger.com/?DOI=446674). Bei Koppers et al. [2011] und Maina et al. [2009] fehlen Angaben $\mathrm{zu}$ weiteren Depressionsbehandlungen im Follow-up, sowohl bezüglich medikamentöser als auch psychotherapeutischer Behandlungen. Dobson et al. [2008] gaben an, dass im 1. Jahr des Followup in den psychotherapeutischen Behandlungsarmen jeweils $1 \mathrm{~Pa}$ tient eine weitere Therapie aufgenommen hatte und deshalb aus der Studie ausgeschlossen worden war.

\section{Erhaltungstherapie}

Eine medikamentöse Erhaltungstherapie während des Followup-Zeitraums wurde bei Dobson et al. [2008] in den ersten 12 Monaten und bei Evans et al. [1992], Maina et al. [2009] und Blackburn et al. [1986] in den ersten 6 Monaten durchgeführt. Nur bei Blackburn et al. [1986] fand neben der medikamentösen auch eine Art psychotherapeutischer Erhaltungstherapie innerhalb der ersten 6 Monate des Follow-up statt. So wurde in den ersten 6 Monaten alle 6 Wochen eine psychotherapeutische Booster-Sitzung angeboten.

\section{Kurzfristige Wirksamkeit von Psychotherapie und medikamentöser Behandlung}

Bei der Mehrzahl der Studien gab es nach der Akuttherapie keinen signifikanten Unterschied zwischen den verschiedenen Behandlungsformen [Beck et al., 1985; Blackburn et al., 1986; De Jong-Meyer et al., 1996; Evans et al., 1992; Koppers et al., 2011; Maina et al., 2009; Mynors-Wallis et al., 2000; Schramm et al., 2007; Shea et al., 1992; Simons et al., 1986]. Dobson et al. [2008], Kovacs et al. [1981] und Weissman et al. [1981] stellten nach der Akuttherapie signifikante Unterschiede im Therapieerfolg zwischen den verschiedenen Behandlungsarmen fest (Online Supplemental Tabelle; $w w w$. karger.com/?DOI=446674).

\section{Langfristige Wirksamkeit von Psychotherapie und medikamentöser Behandlung}

In einigen Vergleichen konnte kein signifikanter Unterschied zwischen Psycho- und Pharmakotherapie im Hinblick auf langfristige Wirksamkeit gefunden werden. Während es aber auch Vergleiche gab, in denen sich die Psychotherapie der Pharmakotherapie (auch als Erhaltungstherapie) überlegen zeigte, gab es keinen Vergleich, der den umgekehrten Fall zeigen konnte. Gleiches gilt für den Vergleich einer alleinigen Psychotherapie/Psychopharmakotherapie mit einer Kombinationsbehandlung (Psychotherapie plus Psychopharmakotherapie). Die Psychotherapie war in allen Vergleichen langfristig ebenso wirksam wie die Kombinationsbehandlung [Beck et al., 1985; Blackburn et al., 1986; Evans et al., 1992; Koppers et al., 2011; Mynors-Wallis et al., 2000; Simons et al., 1986; Weissman et al., 1981]. Bei dem Vergleich einer alleinigen Pharmakotherapie mit einer Kombinationsbehandlung zeigte sich hingegen ein gemischtes Bild. Zwar gab es Vergleiche, in denen die Pharmakotherapie (darunter 1 mit medikamentöser Erhaltungstherapie) ebenso effektiv wie die Kombinationsbehandlung abschnitt [De Jong-Meyer et al., 1996; Evans et al., 1992; Mynors-Wallis et al., 2000; Simons et al., 1986; Weissman et al., 1981], allerdings gab es auch Vergleiche, die auf eine Überlegenheit der Kombinationsbehandlung hinweisen (darunter auch 1 Studie mit medikamentöser Erhaltungstherapie) [Blackburn et al., 1986; Evans et al., 1992; Maina et al., 2009; Schramm et al., 2007; Zobel et al., 2011]. Bei Simons et al. [1986] zeigte sich im Vergleich der Behandlungsarme kein signifikanter Unterschied. In einer weiteren Analyse wurde zwischen den Patienten, denen ein Medikament gegeben wurde (Monotherapie, Kombination mit Psychotherapie), und Patienten, die keines erhielten, unterschieden. Hier zeigten sich signifikant höhere Rückfallraten in der Gruppe mit Medikamenten. In einer weiteren Analyse verglichen die Autoren die Daten von Patienten, die eine Psychotherapie - ob alleinig oder in Kombination - erhielten, mit denen, die keine erhielten, und fanden eine signifikant niedrigere Rückfallrate bei den Patienten mit Psychotherapie. Über alle in diesem Review eingeschlossenen Studien hinweg lagen die Rückfallraten nach Beendigung einer Psychotherapie bei $20-50 \%$, bei einer Psychopharmakotherapie bei $29-78 \%$ und bei einer Kombinationsbehandlung bei 15-44\% (Abb.2). Auch unter den Patienten, die bei Follow-up-Messungen (immer noch) eine Remission oder ein Ansprechen erreichten, war der Anteil der rein pharmakotherapeutisch behandelten Patienten mit 11-35\% bzw. 23-69\% im Vergleich zur Kombinationsbehandlung (28-57\% bzw. 49-71\%) oder Psychotherapie (56-80\% bzw. 34-44\%) kleiner.

\section{Kurzfristige und langfristige Wirksamkeit im Vergleich}

In den meisten Studien ohne Unterscheidung zwischen Psychound Pharmakotherapie oder Kombinationstherapie nach der Akuttherapie zeigte sich nach dem Follow-up ein Unterschied zwischen den Behandlungsarmen, der auf eine bessere langfristige Wirksamkeit der Psychotherapie hinweist. Bei Shea et al. [1992] und De Jong-Meyer et al. [1996] waren auch nach dem Follow-up keine signifikanten Unterschiede zwischen den Behandlungsarmen zu verzeichnen. Bei Dobson et al. [2008] entwickelte sich die als Akuttherapie der medikamentösen Therapie und der Verhaltensaktivierung unterlegene Kognitive Therapie zu einer der medikamentösen Behandlung überlegenen und gegenüber der Verhaltensaktivierung gleichwertigen Therapiemethode. Die Überlegenheit der Kognitiven Therapie gegenüber der medikamentösen Therapie verschwand in der Studie von Kovacs et al. [1981] zum Zeitpunkt der Follow-up-Messung. Bei Weissman et al. [1981] verschwand 


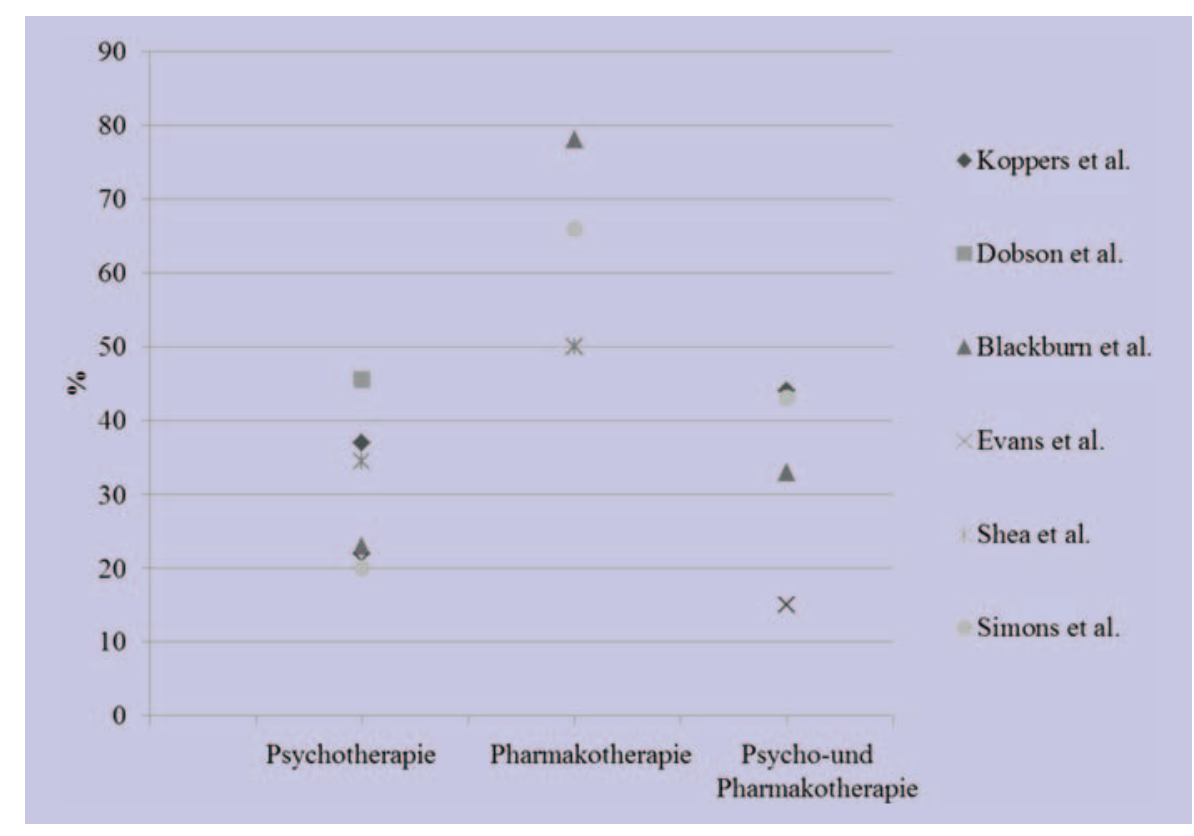

Abb. 2. Rückfallraten im Follow-up nach Beendigung einer Therapie. Schramm et al. [2007] wurde in dieser Abbildung aufgrund der hohen Anzahl an Weiterbehandlungen nach der stationären Akuttherapie nicht dargestellt. die Überlegenheit der Kombinationstherapie gegenüber den einzelnen Monotherapien, sodass alle Behandlungsarme gleich wirksam waren.

\section{Diskussion}

Langfristige Wirksamkeit der Psycho- und Pharmakotherapie in der Depressionsbehandlung

Das vorliegende Review zur langfristigen Wirksamkeit der Psychotherapie liefert 2 Hauptergebnisse: Die bisherige Studienlage deutet darauf hin, dass die Psychotherapie bei mittelschweren unipolaren depressiven Episoden bezüglich der Nachhaltigkeit den Antidepressiva überlegen ist. In keiner Studie war die medikamentöse Therapie - als Akut- oder Erhaltungstherapie - der Psychotherapie überlegen. Während die alleinige Psychotherapie in allen Studien eine vergleichbare Wirksamkeit aufwies wie die Kombinationsbehandlung aus Psycho- und Pharmakotherapie, gab es Studien, in denen eine Unterlegenheit der monotherapeutischen Behandlung mit Antidepressiva festgestellt werden konnte. Es scheint also bezüglich der Kombinationsbehandlung langfristig einen positiven additiven Effekt durch die Psychotherapie, nicht aber durch die Pharmakotherapie zu geben. Dabei scheint die Art der Psychotherapie zweitrangig zu sein, wenngleich mehrheitlich kognitive Verfahren angewandt wurden. Während nach der Akuttherapie größtenteils keine signifikanten Unterschiede zwischen Psychound Pharmakotherapie auftraten, scheinen sich im langfristigen Vergleich Vorteile zugunsten der Psychotherapie und der Kombinationstherapie zu ergeben. Die geringeren Prozentzahlen zu Rückfällen sowie höheren Prozentzahlen zu Therapieerfolgen bei der Psychotherapie im Vergleich zur Pharmakotherapie verdeutlichen ebenso die bessere langfristige Wirksamkeit der Psychotherapie. Die Prozentangaben zu Rückfällen, Ansprechen und Remissionen sind allerdings aufgrund der relativ kleinen Fallzahlen mit
Vorsicht $\mathrm{zu}$ betrachten. Interessant ist auch, dass der Zeitpunkt eines Rückfalls sich nach psychotherapeutischer und medikamentöser Behandlung zu unterscheiden scheint. So zeigte sich bei Evans et al. [1992], dass Rückfälle nach medikamentöser Behandlung schneller auftraten als nach psychotherapeutischen Behandlungen (3,3 Monate; Standardabweichung (SD) 4 Monate vs. 17,4 Monate; SD 12 Monate). Weiterhin gibt es Hinweise, dass Patienten mit medikamentöser Akuttherapie häufiger weitere Behandlungen nach Akuttherapie aufnahmen. Möglicherweise ist dies aber auch durch die höheren Rückfallraten innerhalb dieser Patientengruppe zu erklären.

Als zweites Hauptergebnis ist festzuhalten, dass die Datenlage insgesamt begrenzt ist; so besteht bei dieser für die Praxis hochwichtigen Fragestellung noch ein großer Forschungsbedarf. Zum Einfluss des Schweregrads lässt sich nichts sagen, da dieser Aspekt in den vorliegenden Studien nicht ausreichend differenziert wurde.

\section{Vergleich mit bisherigen Übersichtsarbeiten bezüglich der langfristigen Wirksamkeit}

Wie auch in anderen Übersichtsarbeiten zeigte sich ein reduziertes Rückfallrisiko bei der Psychotherapie im Vergleich zur Pharmakotherapie. Die hier identifizierten Rückfallraten bei der Psychotherapie (20-50\%) und der Pharmakotherapie (29-78\%) passen auch zu den berichteten Rückfallraten zur Psychotherapie bei Gloaguen et al. [1998] (29,5\%), Steinert et al. [2014] (53\%) und Vittengl et al. [2007] (29-54\%) sowie denen zur Psychopharmakotherapie bei Gloaguen et al. [1998] (60\%). In Übereinstimmung mit den Ergebnissen von Karyotaki et al. [2016] und Cuijpers et al. [2014b] erbrachte die kombinierte Behandlung aus Psycho- und Pharmakotherapie langfristig bessere Resultate als die rein medikamentöse Behandlung. Wie auch bei Karyotaki et al. [2016], Steinert et al. [2014] und Cuijpers et al. [2013b] wurden Studien, die über Patienten sowohl mit als auch ohne Therapieerfolg nach Akuttherapie berichteten, eingeschlossen. 


\section{Dauer des Follow-up}

Zwar lag der Follow-Up Zeitraum zwischen 1-6 Jahren, allerdings berichtete die Mehrzahl der Studien nur über Zeiträume von 1 und 2 Jahren. Nur in 3 Studien dauerte das Follow-up länger als 4 Jahre. In 2 dieser Studien [Koppers et al., 2011; Mynors-Wallis et al., 2000] zeigte sich im langfristigen Vergleich kein Unterschied zwischen Psycho- und Pharmakotherapie, jedoch verzeichneten Maina et al. [2009] und Zobel et al. [2011] einen additiven Effekt der Psychotherapie innerhalb einer Kombinationsbehandlung gegenüber einer alleinigen Therapie mit Antidepressiva. Zur Beobachtung von Vittengl et al. [2007], dass ein längerer Follow-upZeitraum auch zu höheren Rückfallraten führt, kann aufgrund der kleinen Studienzahl und der Heterogenität der Studien keine Aussage getroffen werden.

\section{Limitationen der eigenen Studie}

Da nur deutsch- und englischsprachige Literatur eingeschlossen werden konnte, ist es möglich, dass nicht alle relevanten Artikel gefunden wurden. Auch kann nicht ausgeschlossen werden, dass die Suchstrategie nicht optimal war. Um diese Eventualität zu verringern, wurden Literaturverzeichnisse relevanter Studien, Reviews und Meta-Analysen durchsucht.

\section{Limitationen der eingeschlossenen Studien}

Die Ergebnisse dieses Reviews sind allerdings aufgrund einiger Faktoren mit Vorsicht zu betrachten. Zum einen ist methodisch zu bedenken, dass die Datenqualität in klinischen Studien ab dem Behandlungsende in der Regel abnimmt (z.B. mehr fehlende Werte), sodass Wirkungsunterschiede umso schwerer darstellbar sind. Auch gab es zu wenige Studien mit einem Follow-up-Zeitraum von mehr als 2 Jahren, um Aussagen zur langfristigen Wirksamkeit über diesen Zeitraum hinaus treffen zu können. Außerdem ist zu bedenken, dass der Zeitraum der medikamentösen Behandlung bei teilweise nur 3 Monaten lag und somit viel kürzer war als in den Leitlinien empfohlen [APA, 2010; DGPPN et al., 2015; NICE, 2016]. Weiterhin ist kritisch zu betrachten, dass die Anzahl der Psychotherapiesitzungen sehr heterogen war, mit einer Range von 6-30 Sitzungen, was die Vergleichbarkeit der Psychotherapien erschwert. Was genau im Follow-up-Zeitraum passiert, ist bislang nicht standardisiert, wobei eine Standardisierung auch schwer umsetzbar sein dürfte. Es ist also weitestgehend unklar, ob und wie viele weitere psycho- und pharmakotherapeutische Behandlungen während des Follow-up-Zeitraums stattfanden und welchen Einfluss dies auf die Ergebnisse hatte. Eine weitere Limitation der Studien ist der Umgang mit den Begriffen «Rückfall» (relapse), «Ansprechen» (response), «Remission», «Genesung» (recovery), «Rezidiv» (recurrence), «anhaltendes Ansprechen» (sustained response), «anhaltende Remission» (sustained remission) und «anhaltende Genesung» (sustained recovery). Problematisch ist, dass die Begriffe in den Studien größtenteils unterschiedlich - mit mehr oder weniger großen Abweichungen - definiert sind. Schließlich ist ein wichtiger Diskussionspunkt, ob Aussagen zur langfristigen Wirksamkeit nur bei Patienten mit Therapieerfolg nach Akuttherapie getroffen werden können. In 4 Studien wurden nur Patienten mit
Therapieerfolg nach Akuttherapie in das Follow-up eingeschlossen. Für einen solchen Umgang mit den Daten spricht, dass nur so tatsächliche Rückfälle identifiziert werden können. Andererseits entgehen einem möglicherweise so die Effekte der Therapie, die mit einer Latenzzeit verbunden sind.

\section{Implikationen für zukünftige Forschung}

Um fundierte Aussagen über die langfristige Wirksamkeit von Psycho- und Pharmakotherapie in der Depressionsbehandlung treffen zu können, bedarf es weiterer Studien mit längeren Followup-Zeiträumen. Zudem wäre es wünschenswert, die Follow-upZeiträume bezüglich der Aufnahme von weiteren Therapien besser zu dokumentieren. Aber auch eine genauere Dokumentation der tatsächlich erhaltenen Therapieeinheiten, also nicht nur der im Studienprotokoll vorgeschriebenen, wäre wichtig. Um Studienergebnisse besser miteinander vergleichen zu können, bedarf es einer einheitlicheren Definition von Therapieerfolg und Rückfall. Es wäre erstrebenswert, immer beides anzugeben, um die Vergleichbarkeit zwischen Studien zu erhöhen. Ferner bedarf es einer Kombination aus Fremd- und Selbst-Rating bezüglich der Bewertung der Symptomatik, um mögliche Unterschiede zu identifizieren, da die primäre Fokussierung auf Experten-Ratings (z.B. HAMD) Versuchsleiter-Effekte erhöht. Zusätzlich zu Aussagen zur Symptomatik wären auch Daten zum allgemeinen Funktionsniveau und zur Lebensqualität von Interesse. So zeigte sich beispielsweise bei Kovacs et al. [1981], dass durch eine Psychotherapie die Art des Denkens und pessimistische Einstellungen nachhaltig verändert wurden. Zukünftige Studien sollten auch die langfristige Wirksamkeit von Psycho-und Pharmakotherapie im stationären Setting untersuchen. Schließlich ist es von besonderer Wichtigkeit, alle Daten das heißt von Patienten mit und ohne Therapieerfolg sowie von sogenannten ITT- und Completer-Patienten - anzugeben. Die Erfolgs- bzw. Rückfallquoten der eingeschlossenen Studien zeigen, dass es noch Raum für eine Behandlungsoptimierung gibt.

\section{Implikationen für die Praxis}

Das vorliegende Review kommt zu dem Fazit, dass die Psychotherapie bei Depression bezüglich nachhaltiger Effekte einer medikamentösen Behandlung überlegen ist. Die Frage der Nachhaltigkeit von Therapien sollte in den Leitlinien künftig stärker berücksichtigt werden. Zumindest sollte eine Empfehlung dafür gegeben werden, die Betroffenen im Falle einer medikamentösen Therapie besser über das Fehlen nachhaltiger Wirkungen bei Antidepressiva und die Unsicherheit hinsichtlich möglicherweise sogar negativer langfristiger Effekte zu informieren. Eine Studie, die in einem kontrollierten Design untersucht, ob Antidepressiva im Vergleich mit Placebo das Rückfallrisiko nach Beendigung der Einnahme beeinflussen, ist bislang nicht durchgeführt worden. Bisherige Studien, die das Rückfallrisiko nach depressiven Episoden oder das Rezidivrisiko nach Remission nach depressiven Episoden im Vergleich mit Placebo untersuchen, beziehen sich auf Stichproben von Patienten, die bereits vorher Antidepressiva erhalten hatten, sodass unklar bleibt, ob in den jeweiligen Placebogruppen das Rückfall- bzw. Rezidivrisiko durch die vorhergehende Antidepressivagabe beein- 
flusst wurde. Die bestehende Unsicherheit bezüglich langfristiger Wirkungen von Antidepressiva und der hier bestehende Forschungsbedarf sprechen derzeit dafür, bei depressiven Erkrankungen noch stärker die Möglichkeiten der Psychotherapie zu nutzen und die Betroffenen stärker im Sinne einer partizipativen Entscheidungsfindung in die Wahl der jeweiligen Therapieform einzubeziehen.

\section{Online Supplemental Material}

Online Supplemental Tabelle Übersicht der eingeschlossenen Studien To access the supplemental table please refer to $w w w$. karger.com/?DOI=446674.

\section{Disclosure Statement}

Die Autoren erklären, dass kein Interessenkonflikt besteht.

\section{Literatur}

Andrews P, Thomson J, Amstadter A, et al: Primum non nocere: an evolutionary analysis of whether antidepressants do more harm than good. Front Psychol 2012;3:117.

APA: Practice Guideline for the Treatment of Patients With Major Depressive Disorder, ed 3. American Psychiatric Association, 2010.

Babyak M, Blumenthal J, Herman S, et al: Exercise treatment for major depression: maintenance of therapeutic benefit at 10 months. Psychosom Med 2000;62: 633-638.

Beck A, Hollon S, Young J, et al: Treatment of depression with cognitive therapy and amitriptyline. Arch Gen Psychiatry 1985;42:142-148.

Blackburn I, Eunson K, Bishop S: A two-year naturalistic follow-up of depressed patients treated with cognitive therapy, pharmacotherapy and a combination of both J Affect Disord 1986;10:67-75.

Cuijpers P, Andersson G, Donker T, et al: Psychological treatment of depression: results of a series of metaanalyses. Nord J Psychiatry 2011a;65:354-364.

Cuijpers P, Berking M, Andersson G, et al: A meta-analysis of cognitive-behavioural therapy for adult depression, alone and in comparison with other treatments. Can J Psychiatry 2013a;58:376-385.

Cuijpers P, Geraedts A, van Oppen P, et al: Interpersonal psychotherapy for depression: a meta-analysis. Am J Psychiatry 2011b;168:581-592.

Cuijpers P, Hollon S, van Straten A, et al: Does cognitive behaviour therapy have an enduring effect that is superior to keeping patients on continuation pharmacotherapy? A meta-analysis. BMJ Open 2013b;3:e002542.

Cuijpers P, Karyotaki E, Weitz E, et al: The effects of psychotherapies for major depression in adults on remission, recovery and improvement: A meta-analysis. J Affect Disord 2014a;159:118-126.

Cuijpers P, Sijbrandij M, Koole SL, et al: Adding psychotherapy to antidepressant medication in depression and anxiety disorders: a meta-analysis. World Psychiatry 2014b;13:56-67.

De Jong-Meyer R, Hautzinger M, Rudolf G, et al: Die Überprüfung der Wirksamkeit einer Kombination von Antidepressiva- und Verhaltenstherapie bei endogenen depressiven Patienten: Varianzanalytische Ergebnisse zu den Haupt-und Nebenkriterien des Therapieerfolges. Z Klin Psychol 1996;25:93-109.

DGPPN, BÄK, KBV, et al: S3-Leitlinie/Nationale VersorgungsLeitlinie Unipolare Depression - Langfassung, ed 2, Version 2. 2015
Dobson K, Hollon S, Dimidjian S, et al: Randomized trial of behavioral activation, cognitive therapy, and antidepressant medication in the prevention of relapse and recurrence in major depression. J Consult Clin Psychol 2008;76:468-477.

Driessen E, Hegelmaier L, Abbass A, et al: The efficacy of short-term psychodynamic psychotherapy for depression: A meta-analysis update. Clin Psychol Rev 2015; 42:1-15.

Eaton WW, Shao H, Nestadt G, et al: Population-based study of first onset and chronicity in major depressive disorder. Arch Gen Psychiatry 2008;65:513-520.

Evans M, Hollon S, DeRubeis R, et al.: Differential relapse following cognitive therapy and pharmacotherapy for depression. Arch Gen Psychiatry 1992;49:802-808.

Gloaguen V, Cottraux J, Cucherat M, et al: A meta-analysis of the effects of cognitive therapy in depressed patients. J Affect Disord 1998;49:59-72.

Karyotaki E, Smit Y, Holdt Henningsen K, et al: Combining pharmacotherapy and psychotherapy or monotherapy for major depression? A meta-analysis on the long-term effects. J Affect Disord 2016;194:144-152.

Kessler R, Berglund P, Demler O, et al: The epidemiology of major depressive disorder: results from the National Comorbidity Survey Replication (NCS-R). JAMA 2003;289:3095-3105.

Khan A, Faucett J, Lichtenberg P, et al: A systematic review of comparative efficacy of treatments and controls for depression. PLoS ONE 2012;7:e41778.

Kirsch I: Der Placeboeffekt in der antidepressiven Behandlung. Verhaltenstherapie 2016;26:55-61.

Koppers D, Peen J, Niekerken S, et al: Prevalence and risk factors for recurrence of depression five years after short term psychodynamic therapy. J Affect Disord 2011;134:468-472.

Kovacs M, Rush A, Beck A, et al: Depressed outpatients treated with cognitive therapy or pharmacotherapy. A one-year follow-up. Arch Gen Psychiatry 1981;38:3339

Maina G, Rosso G, Bogetto F: Brief dynamic therapy combined with pharmacotherapy in the treatment of major depressive disorder: long-term results. J Affect Disord 2009;114:200-207.

Marschall J, Hildebrandt S, Sydow H, et al: Gesundheitsreport 2016: Analyse der Arbeitsunfähigkeitsdaten. Beiträge zur Gesundheitsökonomie und Versorgungsforschung, Band 13. Heidelberg, medhochzwei Verlag, 2016.
Murphy J, Byrne G: Prevalence and correlates of the proposed DSM-5 diagnosis of Chronic Depressive Disorder. J Affect Disord 2012;139:172-180.

Mynors-Wallis L, Gath D, Day A, et al: Randomised controlled trial of problem solving treatment, antidepressant medication, and combined treatment for major depression in primary care. BMJ 2000;320:26-30

NICE: Depression in adults: recognition and management. NICE guidelines (CG90). National Institute for Health and Clinical Excellence, 2016.

Schramm E, van Calker D, Dykierek P, et al: An intensive treatment program of interpersonal psychotherapy plus pharmacotherapy for depressed inpatients: acute and long-term results. Am J Psychiatry 2007;164:768-777.

Shea M, Elkin I, Imber S, et al: Course of depressive symptoms over follow-up. Findings from the National Institute of Mental Health Treatment of Depression Collaborative Research Program. Arch Gen Psychiatry 1992;49:782-787.

Shrestha S, Nelson E, Liow J, et al: Fluoxetine administered to juvenile monkeys: effects on the serotonin transporter and behavior. Am J Psychiatry 2014;171:323-331.

Simons A, Murphy G, Levine J, et al: Cognitive therapy and pharmacotherapy for depression. Sustained improvement over one year. Arch Gen Psychiatry 1986;43:4348.

Steinert C, Hofmann M, Kruse J, et al: Relapse rates after psychotherapy for depression - stable long-term effects? A meta-analysis. J Affect Disord 2014;168:107-118.

Vittengl J, Clark L, Dunn T, et al: Reducing relapse and recurrence in unipolar depression: a comparative metaanalysis of cognitive-behavioral therapy's effects. J Consult Clin Psychol 2007;75:475-488.

Weissman M, Klerman G, Prusoff B, et al: Depressed outpatients. Results one year after treatment with drugs and/or interpersonal psychotherapy. Arch Gen Psychiatry 1981;38:51-55.

Weitz E, Hollon S, Twisk J, et al: Baseline Depression Severity as Moderator of Depression Outcomes Between Cognitive Behavioral Therapy vs Pharmacotherapy: An Individual Patient Data Meta-analysis. JAMA Psychiatry 2015;72:1102-1109.

Zobel I, Kech S, van Calker D, et al: Long-term effect of combined interpersonal psychotherapy and pharmacotherapy in a randomized trial of depressed patients. Acta Psychiatr Scand 2011;123:276-282. 